\title{
A Computer-Assisted Non-Image-Based Method for Locating the Center of Femoral Head
}

\author{
Jian Wu and Ang Li
}

\begin{abstract}
In total knee replacement (TKR) operation, a key factor for successful operation is to locate the center of the femoral head accurately, and to determine the mechanical axis, which goes through the center of femoral head and knee joint. Place an infrared tracker on the distal end of the femur, rotate the bone and then track the movement with the tracker, thus the center of the femoral head can be determined. We use the Hough transform in this study to convert the least square calculation into a dualistic linear regression, therefore the algorithm is optimized and the computing time is reduced. We also use RANdom SAmple Consensus (RANSAC) algorithm for data screening, which has enhanced the method's resistance to interference during the surgery. Combining the two advantages, this method can reach low deviation within $2 \mathrm{~mm}$, fast computing time less than $0.5 \mathrm{~s}$, despite of interferences.
\end{abstract}

Index Terms-Locating femoral center, infrared locator, hough transform, RANSAC.

\section{INTRODUCTION}

Knee joint is the most frequently injured joint of the human body. Currently, the TKR operationis an effective way to cure severe knee joint injury. Patients after TKR treatment can recover physical capability to a certain extent. In a TKR operation, defining the mechanic axis of the lower limbs is a very important procedure. The mechanic axis goes through the center of femoral head and knee joint. A slight deviation in the mechanic axis can lead to post-procedure aches and shorten the life of the prosthesis.

The purpose of this study is to find a method to determine the mechanic axis of the lower limbs by locating the center of the femoral head [1]. It is both the anatomical center and kinematic center. Traditional ways of locating has significant deviation, later, researchers used infrared tracker to locate the center of femoral head instead [2], [3]. The traditional method was put forward in 1997, by Leitner et al. [4], who used a tracker at the distal end of the femur to locate the center of the femoral head. Nonetheless, this study proposed a method to further process the data collected via the previous approach, through Hough transform, this method can transform the least square calculation into a dualistic linear regression, therefore simplified the computing process. Then

Manuscript received March 11, 2013; revised May 17, 2013. This work was supported in part by National Natural Science Foundation (81000649), SZ-HK innovation circle 'Computer Assisted TKR surgery key technology and post-procedure rehabilitation research project'.

The authors are with the Shenzhen Key Laboratory for Minimally Invasive Medical Technologies, Graduate School at Shenzhen, Tsinghua University, Shenzhen 518055 China (tel: +86-755-26036353; fax: +86-755260353; e-mail: wuj@sz.tsinghua.edu.cn,bmelionel@gmail.com). this method used RANSAC [5] algorithm to screen the data, this also improved the method's resistance to interferences.

\section{METHOD}

We collect data by using the Leitner`s method, as Fig. 1 shows; however, this method has low resistance to data interference. Therefore this study has introduced RANSAC algorithm to improve its resistance to interference; on the other hand, the algorithm also reduced the computing speed. Then Hough transform is applied to transform the least square calculation into a linear regression, in order to shorten the computing time. This study proposed a method to combine these two techniques, to get a high speed, accurate, and interference-resilient algorithm.

\section{A. Spherical Surface Fitting}

The hip joint (coxa joint) includes the femoral head and the acetabular fossa; when the femur moves, any point on the femur would move on a spherical surface, the center of the sphere is the center of the femoral head. Leitner's method suggests that if an infra-red locater is put on the distal femur (the end close to tibia), then rotate the femur, we can use the locator to track the movement [4], [6]. If 4 non-coplanar points can be tracked, then the center of this sphere can be calculated using the following formula:

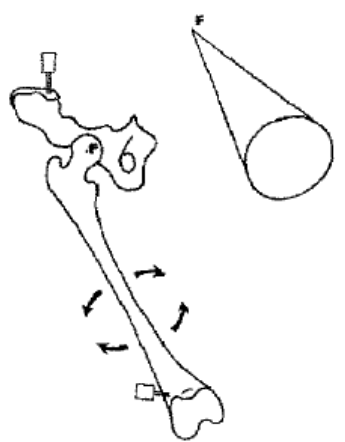

Fig. 1. Illustration of the experiment [4].

$\left\{\begin{array}{l}\left(x_{o}-x_{1}\right)^{2}+\left(y_{o}-y_{1}\right)^{2}+\left(z_{o}-z_{1}\right)^{2}=\left(x_{o}-x_{2}\right)^{2}+\left(y_{o}-y_{2}\right)^{2}+\left(z_{o}-z_{2}\right)^{2} \\ \left(x_{o}-x_{1}\right)^{2}+\left(y_{o}-y_{1}\right)^{2}+\left(z_{o}-z_{1}\right)^{2}=\left(x_{o}-x_{3}\right)^{2}+\left(y_{o}-y_{3}\right)^{2}+\left(z_{o}-z_{3}\right)^{2} \\ \left(x_{o}-x_{1}\right)^{2}+\left(y_{o}-y_{1}\right)^{2}+\left(z_{o}-z_{1}\right)^{2}=\left(x_{o}-x_{4}\right)^{2}+\left(y_{o}-y_{4}\right)^{2}+\left(z_{o}-z_{4}\right)^{2}\end{array}\right.$
$\left(x_{i}, y_{i}, z_{i}\right)$ is the center of the sphere, $\left(x_{1}, y_{1}, z_{1}\right)$, $\left(x_{2}, y_{2}, z_{2}\right),\left(x_{3}, y_{3}, z_{3}\right),\left(x_{4}, y_{4}, z_{4}\right)$ are coordinates tracked by the infra-red locator. Convert these data into a matrix:

$$
T_{1}=\left[\begin{array}{lll}
2\left(x_{2}-x_{1}\right) & 2\left(y_{2}-y_{1}\right) & 2\left(z_{2}-z_{1}\right) \\
2\left(x_{3}-x_{1}\right) & 2\left(y_{3}-y_{1}\right) & 2\left(z_{3}-z_{1}\right) \\
2\left(x_{4}-x_{1}\right) & 2\left(y_{4}-y_{1}\right) & 2\left(z_{4}-z_{1}\right)
\end{array}\right]
$$




$$
T_{2}=\left[\begin{array}{l}
\left(x_{2}^{2}-x_{1}^{2}\right)+\left(y_{2}^{2}-y_{1}^{2}\right)+\left(z_{2}^{2}-z_{1}^{2}\right) \\
\left(x_{3}^{2}-x_{1}^{2}\right)+\left(y_{3}^{2}-y_{1}{ }^{2}\right)+\left(z_{3}^{2}-z_{1}^{2}\right) \\
\left(x_{4}^{2}-x_{1}^{2}\right)+\left(y_{4}{ }^{2}-y_{1}{ }^{2}\right)+\left(z_{4}{ }^{2}-z_{1}^{2}\right)
\end{array}\right]
$$

Thus, the coordinates of the sphere center: $O\left(x_{o}, y_{o}, z_{o}\right)$

$$
\left[\begin{array}{c}
x_{o} \\
y_{o} \\
z_{o}
\end{array}\right]=T_{1}^{-1} T_{2}
$$

However, in real-life practices, more than four input coordinates are needed for computing the sphere center, usually dozens of coordinates are needed. Though the sphere center can be obtained by computing the mean value of all the data, a more reasonable means is to use the least square method, to compute the coordinates of the center point, and the function is as follow:

$$
f\left(x_{o}, y_{o}, z_{o}, r\right)=\sum_{i=1}^{N}\left(\sqrt{\left(x_{i}-x_{o}\right)^{2}+\left(y_{i}-y_{o}\right)^{2}+\left(z_{i}-z_{o}\right)^{2}}-r\right)
$$

$N$ is the number of collected data, $r$ is the distance between the infrared-locator and the center of the femoral head. $x_{i}, y_{i}, z_{i}$ are the spatial coordinates of the locator; $x_{o}, y_{o}, z_{o}$ are the coordinates of the center of the femoral head.

In order to get the minimum of the function, the partial derivative of the four parameters should be assumed to be 0 :

$$
\frac{\partial f}{\partial x_{o}}=\frac{\partial f}{\partial y_{o}}=\frac{\partial f}{\partial z_{o}}=\frac{\partial f}{\partial r}=0
$$

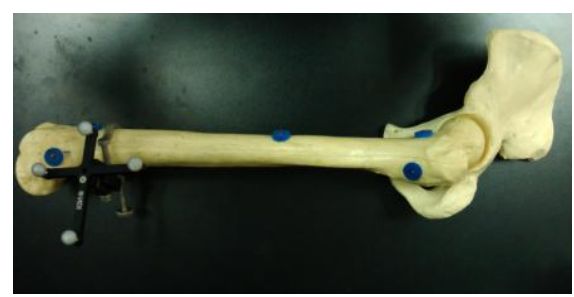

Fig. 2. Our experimental facility.

\section{B. Hough Transform}

The conventional method requires a fitting using non-linear least square calculation; but due to the complexity of this function, using this method is very time-consuming, also if real-time measurement is required, the time-effectiveness would also be compromised. As a result, our study introduced a method based on Hough transform, to transform the least square calculation into a dualistic linear regression. It is done via the following steps:

Chose any two points on the sphere surface, $A\left(x_{1}, y_{1}, z_{1}\right), B\left(x_{2}, y_{2}, z_{2}\right)$, locate the mid-point of line segment $\mathrm{AB}$, its coordinates are: $\left(\frac{x_{1}+x_{2}}{2}, \frac{y_{1}+y_{2}}{2}, \frac{z_{1}+z_{2}}{2}\right)$ and its vector $\overrightarrow{A B}:\left(x_{1}-x_{2}, y_{1}-y_{2}, z_{1}-z_{2}\right)$. Using $\overrightarrow{A B}$ as a normal vector, to determine the perpendicular bi-sector plane of $A B$, the formula of the normal point is as follow:

$$
\left(x_{1}-x_{2}\right)\left(x-\frac{x_{1}+x_{2}}{2}\right)+\left(y_{1}-y_{2}\right)\left(y-\frac{y_{1}+y_{2}}{2}\right)+\left(z_{1}-z_{2}\right)\left(z-\frac{z_{1}+z_{2}}{2}\right)=0
$$

Simplify (7):

$$
x+B y+C z+D=0
$$

Thus this plane in the variable space is named as $\alpha$.

1) Because point $A$ and $B$ are on the sphere surface, so the center of the sphere must locate on the plane $\alpha$, which splits the line segment $\mathrm{AB}$, therefore:

$$
x_{0}+B y_{0}+C z_{0}+D=0
$$

2) Using Hough transform, the plane $\alpha$ in the variable space $(x, y, z)$ is transformed into the parameter space $(B, C, D)$ as plane: $D+y_{0} B+z_{0} C+x_{0}=0$. Actually the planes will not intersect exactly at one point considering the measurement errors, but the intersections may be very close to each other.

3) For there are multiple planes like $\alpha$, these planes should intersect at a point $O\left(x_{o}, y_{o}, z_{o}\right)$, which is the center point of the sphere; therefore, in the parameter space $(B, C, D)$, there should be multiple points which can be determined by parameter $(B, C, D)$, and all these points are located on a spherical surface defined by the spherical center $O\left(x_{o}, y_{o}, z_{o}\right)$.

Based on the calculation above, then apply dualistic linear regression to the data, the coordinates of the spherical center $O\left(x_{o}, y_{o}, z_{o}\right)$ can be determined.

\section{RANSAC Data Screening}

Due to varying conditions of the exam and the shock during the surgery, erroneous or invalid data maybe collected along with useful data. The Leitner's method did not contain a method to eliminate abnormal data, that means, the collected data is supposed to meet with the smoothness assumption: the size of data set is ignored, and assumed that there are always enough correct data to make up for the impact of abnormal data. But in real practice, there may be a large amount of erroneous data, which would cause errors and make it impossible to calculate via formula fitting.

To solve this problem, this study used data screening to rule out abnormal data; the basic idea of RANSAC data screening is as follow:

1) From the collected data, a random selection of points are picked out; these points are assumed to be valid data; then these data are used in parameter fitting to form a model.

2) The model acquired in step 1 is then used to evaluate other data; if the distance between a data point and the model has reached a certain threshold, then the point is judged also as valid, otherwise it would be judged as invalid.

3) Now use all the new data from step 2 to do parameter fitting again, and generate a new model.

4) Repeat step 1 through 3 , a new model is generated, then 
the new model is compared with the old one, if the new model contains more valid data, it will be considered as better, and will replace the old one; if not, the new model will be abandoned.

After repeating this process for a certain time, it can be assumed that the last model remained after the loop is the best one in the time given.

\section{EXPERIMENT AND RESULTS}

This experiment used the Polaris Spectra System infra-red tracker, manufactured by NDI, to calculate the center of the femur.

Fig. 3 shows the tracked dots which are distributed on the sphere, whose center is also shown on the figure (unit: $\mathrm{mm}$ ):

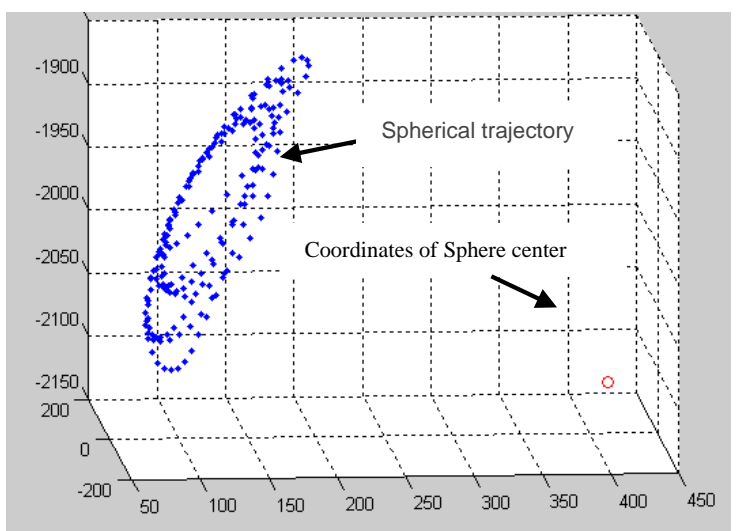

Fig. 3. The dots resemble the movement of the femur on a spherical surface.

Turn the femur 20 times, and collected the following data:

TABLE I: RESULTS GENERATED USING 4 DIFFERENT ALGORITHMS (WITHOUT INTERFERENCE)

\begin{tabular}{lll}
\hline \hline Method & Mean Deviation $(\mathrm{mm})$ & Mean Time $(\mathrm{s})$ \\
\hline Traditional & 0.74 & 0.10 \\
Traditional RANSAC & 0.94 & 3.57 \\
HoughTransform & 1.27 & 0.009 \\
HoughTransform+RANSAC & 1.99 & 0.450 \\
\hline \hline
\end{tabular}

TABLE II: CALCULATION RESULTS OF 4 ALGORITHMS (WITH INTERFERENCE)

\begin{tabular}{lll}
\hline Method & Mean Deviation $(\mathrm{mm})$ & Mean Time(s) \\
\hline Traditional & 8.87 & 0.091 \\
Traditional RANSAC & 1.33 & 5.410 \\
HoughTransform & 203.59 & 0.017 \\
HoughTransform+RANSAC & 1.93 & 0.441 \\
\hline \hline
\end{tabular}

Interfered data are intentionally added to $10 \%$ of the data in each group, that is: in every dimension coordinate, a random number with $\pm 10 \mathrm{~mm}$ deviation was added to simulate the shake and vibration which exist in real-life exams. Now with interference, the results are shown in the Table II:

It is obvious that when there is no interference, the results of least square fitting and linear regression are both correct. Assume the length of the femur is $400 \mathrm{~mm}$, the deviation observed in this experiment is $2 \mathrm{~mm}$, which is only $0.29^{\circ}$ deviation from the mechanic axis. Also, the runtime of both methods are short enough to meet the requirement of real-time navigation.

However, when interference was added, the deviation raised significantly; especially the linear regression method was heavily impacted, because the fitting process was done in the parameter space, so when the deviation from the parameter space is transferred to deviation in the variable space, it could generate a deviation as large as $200 \mathrm{~mm}$. Also, using least square method would generate an $8 \mathrm{~mm}$ deviation, which was not accurately enough by surgery standard. To improve resilience to interference, the RANSAC algorithm must be included.

Including the RANSAC algorithm would not heavily affect the accuracy of the fitting calculation; moreover, even under interference, the RANSAC algorithm still can achieve high accuracy as good as when there was no interference. In real-life exams, interference is inevitable, so the algorithm must stand to interference, therefore RANSAC algorithm is very important in this calculation.

A disadvantage of RANSAC algorithm is, it will prolong the time for calculation, because it contains a lot of iteration and fitting. As the result shows, using RANSAC algorithm with least square method, would cost 3 to 6 seconds, this will pose a large impact on real-time measurements. As a result, linear regression is used to improve the speed of calculation. Using linear regression and RANSAC together would only cost less than $1 / 2$ second to do the computation.

In the experiment, we measured the real coordinate of center of femoral head through a group of CT data (showed in Fig. 4) [7], [8]. Table III shows an example of our measurement. The NDI track system has a RMS error around $0.25 \mathrm{~mm}$.

TABLE III: AN EXAMPLE OF MEASUREMENT

\begin{tabular}{lcl}
\hline \hline Method & Coordinate $(\mathrm{mm})$ & Error $(\mathrm{mm})$ \\
\hline CT data & $(-49.3,6.2,-1934.0)$ & N/A \\
Traditional & $(-44.3,4.8,-1935.8)$ & 5.50 \\
Traditional RANSAC & $(-49.7,5.2,-1934.6)$ & 1.23 \\
HoughTransform & $(-195.1,-9.7,-1895.7)$ & 151.58 \\
HoughTransform+RANSAC & $(-48.6,5.2,-1935.1)$ & 1.64 \\
\hline \hline
\end{tabular}

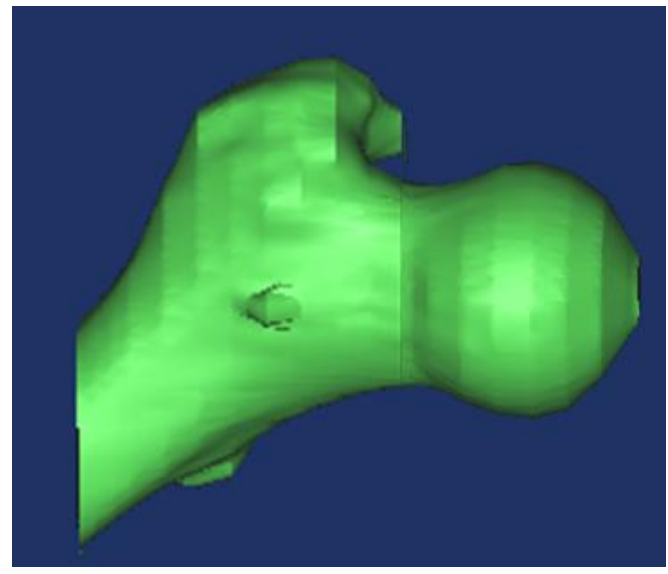

Fig. 4. CT of the femoral head

\section{Discussion}

When calculating the coordinates of the spherical center, the traditional method aims to find the minimal sum of the distance of all data spot to the sphere surface; while in the Hough transform, it aims to calculate the minimal sum of the distance between the sphere center and all the bi-sector planes, after found the entire perpendicular bi-sector plane. These two methods are equally feasible. But when Hough 
transform is applied to the perpendicular bi-sector plane, the calculation actually transformed to compute the minimal sum of all the dots on the perpendicular plane to the plane defined by the spherical center in the parameter space.

The distance between spherical center and the perpendicular bisector plane is:

$$
\frac{x_{0}+B y_{0}+C z_{0}+D}{\sqrt{1+B^{2}+C^{2}}}
$$

Considering the measurement errors, the distance between the perpendicular bisector plane and the plane defined by the spherical center is:

$$
\frac{x_{0}+B y_{0}+C z_{0}+D}{\sqrt{1+y_{0}^{2}+z_{0}^{2}}}
$$

Because $x_{o}, y_{o}, z_{o}$ are stable, so the difference is mostly caused by parameter B, C, D. When the perpendicular plane lies in vertical or parallel position to the coordinate axis, the value of $\sqrt{1+B^{2}+C^{2}}$ may become very large, and thus pose a large deviation on the results. So in the calculation, this kind of data points should be eliminated to ensure accuracy.

This study has proposed a new non-imaging-based method to locate the center of femur head. The new method used Hough transform to simplify the calculation and improved calculation speed. RANSAC data screening is applied to improve resilience to interference. The experiment results show that the new method is both more accurate and more time-saving, it can provide strong support to TKR operations and can be used in surgery navigation systems.

\section{ACKNOWLEDGEMENTS}

Thanks to the support from the following entities and projects: National Natural Science Foundation (81000649), SZ-HK innovation circle 'Computer Assisted TKR surgery key technology and post-procedure rehabilitation research project', Shenzhen Key-lab of Non-invasive Monitoring and Minimal Invasive Medicine Technology.

\section{REFERENCES}

[1] D. Kendoff, T. N. Board, M. Citak, M. J. Gardner, S. Hankemeier, S. Ostermeier, C. Krettek, and T. Hufner, "Navigated lower limb axis measurements: Influence of mechanical weight-bearing simulation," J Orthop Res, vol. 26, pp. 553-561, 2008.

[2] S. M. Martelli, M. Marcacci, L. Nofrini, F. L. Palombara, A. Malvisi, F. Iacono, P. Vendruscolo, and M. Pierantoni, "Computer- and robot-assisted total knee replacement: analysis of a new surgical procedure," Annals of Biomedical Engineering, vol. 28, pp. 1146-1153, 2000.

[3] G. R. Klein, M. S. Austin, E. B. Smith, and W. J. Hozack, "Total knee arthroplasty using computer-assisted navigation in patients with deformities of the femur and tibia," J Arthroplasty, vol. 21, pp. 284-288, 2006.

[4] F. Leitner, F. Picard, R. Minfelde, H. J. Schulz, P. Cinquin, and D. Saragaglia, "Computer-assisted knee surgical total replacement," In Lecture Notes in Computer Science, Grenoble, France, pp. 629, 1997.

[5] M. A. Fischler and R. C. Bolles, "RANdom SAmple Consensus: paradigm for model fitting with applications to image analysis and automated cartography," Communications of the ACM, vol. 24, pp. 381-395, 1981

[6] N. Lopomo, L. Sun, S. Zaffagnini, G. Giordano, and M. R. Safran, "Evaluation of formal methods in hip joint center assessment," An in vitro analysis. Clinical Biomechanics, vol. 25, pp. 206-212, 2010.

[7] M. Kang, "Hip joint center location by fitting conchoid shape to the acetabular rim region of MR Images," In Annual International Conference of the IEEE Engineering in Medicine and Biology Proceedings, San Francisco, CA, United states, pp. 4477-4480, 2004.

[8] V. Sholukha, T. Chapman, P. Salvia, F. Moiseev, F. Euran, M. Rooze and J. S. V. Sint, "Femur shape prediction by multiple regression based on quadric surface fitting," J Biomech, vol. 44, pp. 712-718, 2011.

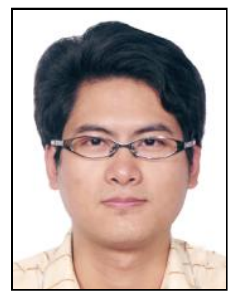

Jian Wu received the doctor degree in biomedical engineering from the Tsinghua University, Beijing, China, in 2004. Since 2006, he has been with the Research Center of Biomedical Engineering, Graduate School at Shenzhen, Tsinghua University, Shenzhen, China, where he has been researching biomedical measurement, computer aided surgery, in particular optical tracking technology. Since 2007, he has been an Associate Professor of biomedical engineering. 